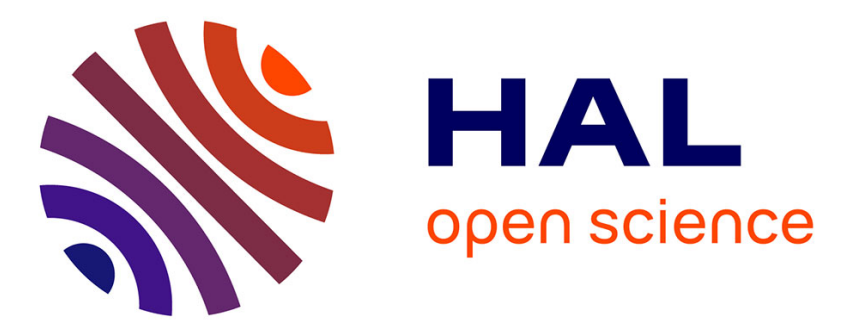

\title{
Hybrid Frequency and Phase-Shift Keying Modulation for Energy Efficient Optical Wireless Systems
}

\author{
Ali Waqar Azim, Yannis Le Guennec, Laurent Ros
}

\section{To cite this version:}

Ali Waqar Azim, Yannis Le Guennec, Laurent Ros. Hybrid Frequency and Phase-Shift Keying Modulation for Energy Efficient Optical Wireless Systems. IEEE Wireless Communications Letters, 2019, pp.1-4. 10.1109/LWC.2019.2954832 . hal-02383633

\section{HAL Id: hal-02383633 \\ https://hal.science/hal-02383633}

Submitted on 27 Nov 2019

HAL is a multi-disciplinary open access archive for the deposit and dissemination of scientific research documents, whether they are published or not. The documents may come from teaching and research institutions in France or abroad, or from public or private research centers.
L'archive ouverte pluridisciplinaire HAL, est destinée au dépôt et à la diffusion de documents scientifiques de niveau recherche, publiés ou non, émanant des établissements d'enseignement et de recherche français ou étrangers, des laboratoires publics ou privés. 


\title{
Hybrid Frequency and Phase-Shift Keying Modulation for Energy Efficient Optical Wireless Systems
}

\author{
Ali Waqar Azim, Yannis Le Guennec, and Laurent Ros
}

\begin{abstract}
In this letter, we introduce direct-current (DC) offset hybrid frequency and phase-shift keying (DC-FPSK) modulation for Internet-of-Things based on optical wireless systems. For DCFPSK, non-negative phase-modulated frequency waveforms are generated by combining frequency-shift keying (FSK), phase-shift keying (PSK) and a DC offset. We propose optimal maximum likelihood and sub-optimal receivers for DC-FPSK. The performance is appraised in terms of Euclidean distance, bit-errorrate (BER) performance and energy efficiency. We determine that combining 4-PSK with conventional DC-FSK is the optimal approach to enhance the energy and spectral efficiencies.
\end{abstract}

Index Terms-Optical wireless system, Internet-of-Things, frequency-shift keying, phase-shift keying, intensity modulationdirect detection.

\section{INTRODUCTION}

$\mathbf{T}$ HE Internet-of-Things (IoT) paradigm expects wireless connectivity for a wide range of devices and objects via the internet. However, the exiguous radio-frequency (RF) spectral resources may contribute to practical limitations in IoT deployment. To this effect, integrating optical wireless systems (OWS) with IoT may alleviate the spectral scarcity dilemma. Consequently, the design of modulation formats for ubiquitous wireless connectivity under low-power scenarios for OWS IoT is of pivotal significance. High energy efficiency (EE) requirement and intensity-modulation/directdetection (IM/DD) implementation of OWS lessen the number of such protocols. Linear modulations, such as $M$-ary pulse amplitude modulation (PAM) and its special case when $M=2$, i.e., on-off keying (OOK) can have forthright implementation for OWS. Nevertheless, increasing alphabet cardinality for linear modulations (e.g., PAM) increases the required electrical/optical energy per bit (respectively denoted by $E_{\mathrm{b}_{\text {(elec) }}}$ and $\left.E_{\mathrm{b}_{(\text {opt }}}\right)$ for a given bit-error-rate (BER). In a channel with additive white Gaussian noise (AWGN) having mono-lateral power spectral density, $N_{0}$, the most energy efficient linear modulation is OOK. Orthogonal modulations, on the other hand, may achieve high EE by sacrificing the spectral efficiency (SE) (done by increasing the alphabet cardinality, $M$ ). $M$-ary pulse position modulation (PPM) [1] and $M$-ary frequency-shift keying (FSK) [2] belong to the class of orthogonal modulations. For OWS, $M$-ary PPM lacks practicality because of high peak-to-average power ratio (PAPR), high peak-to-mean optical power ratio (PMOPR), higher sensitivity to multipath propagation, complex equalization and possible synchronization issues at the receiver [3], [4]. $M$-ary FSK circumvents these limitations, but, is

The authors are with Université Grenoble Alpes, CNRS, Institute of Engineering, Grenoble INP, GIPSA-LAB, 38000 Grenoble, France (email: \{aliwaqar.azim,yannis.le-guennec,laurent.ros\}@ grenoble-inp.fr). bipolar in nature. However, introduction of direct-current (DC) offset to conventional $M$-ary FSK may proceed in IM/DD compatible variant, referred to as $M$-ary DC-FSK [5]. $M$ ary DC-FSK is capable of attaining high EE compared to OOK and asymmetric FSK (AFSK) [6] by relinquishing the SE. Both $M$-ary PPM and $M$-ary DC-FSK possess SE of $\log _{2}(M) / M$ bits/s/Hz. However, only a change in $M$ modifies the SE of the aforementioned approaches. From a simpler perspective, inculcation of an additional degree of freedom (DoF) in modifying the SE can either relax the constraint on the bandwidth, or a higher data-rate can be achieved for a fixed bandwidth. This flexibility could be of interest to address different demands of data-rate/EE for IoT [7].

In this letter, we introduce DC-FPSK modulation based on combination of $M$-ary DC-FSK and phase-shift keying (PSK). Unlike $M$-ary DC-FSK, the SE of DC-FPSK changes with the number of available frequencies and phase-shifts. DC-FPSK is in line with [8], however, the uniqueness lies in the following attributes: (i) it takes into account the non-negativity constraint of IM/DD; (ii) an optimal receiver configuration according to maximum likelihood (ML) criterion is developed; (iii) a reduced complexity sub-optimal receiver is presented; and (iv) closed-form expressions for the squared Euclidean distance are derived. We shall establish that DC-FPSK is superior than DCFSK in terms of SE and EE trade-off. Moreover, we foresee that DC-FPSK can have applications for connected vehicles, indoor localization and tracking, etc. The rest of the article is organized as follows. Section II examines DC-FPSK waveform generation, recovery and its correlation properties. Section III presents analytical and simulation results and conclusions are rendered in section IV.

\section{WAVEFORM GENERATION AND RECOVERY}

In the sequel, we shall refer to the proposed approach as $\left(M_{\perp}, M_{\varphi}\right)$-DC-FPSK, where $M_{\perp}$ and $M_{\varphi}$ are the number of (positive) discrete frequencies and phase-shifts, respectively.

\section{A. Waveform Generation}

$\left(M_{\perp}, M_{\varphi}\right)$-DC-FPSK waveform corresponding to the $m$ th frequency and $i$ th phase composed of alternating-current (AC) and DC components is given as:

$$
s_{m, i}(t)=A \cos \left(2 \pi m \Delta f t+\varphi_{i}\right)+\beta, \quad t \in\left[0, T_{\mathrm{s}}\right],
$$

where $A$ is the amplitude of the AC component. Since it is impossible to modulate the phase of the waveform corresponding to $m=0$, thereby, we consider $m \in\left\{1,2, \cdots, M_{\perp}\right\}$. $\varphi_{i}$ is the phase-shift drawn from the set $\left\{2 \pi i / M_{\varphi}\right\}$ with $i \in\left\{0,1, \cdots, M_{\varphi}-1\right\} . \Delta f=1 / T_{\mathrm{s}}$ is the spectral separation 


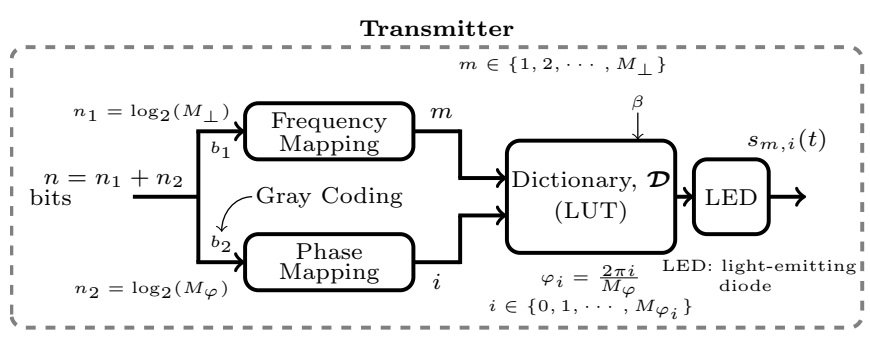

Fig. 1: Transmitter configuration of $\left(M_{\perp}, M_{\varphi}\right)$-DC-FPSK.

within two adjacent frequencies, where $T_{\mathrm{s}}$ is the symbol duration. The single-sided bandwidth is $B=M_{\perp} \Delta f$. Furthermore, the DC offset, $\beta$, when equal to $A$ assures non-negativity of the transmit waveforms. The frequency and phase are chosen via $n_{1}$ bits and Gray coded $n_{2}$ bits, respectively. The electrical symbol energy of a $\left(M_{\perp}, M_{\varphi}\right)$-DC-FPSK waveform is

$$
E_{\mathrm{s}(\text { elec })}=\int_{0}^{T_{\mathrm{s}}} s_{m, i}^{2}(t) \mathrm{d} t=\left(\frac{A^{2}}{2}+\beta^{2}\right) T_{\mathrm{s}}=\frac{3 A^{2}}{2} T_{\mathrm{s}},
$$

which is independent of the frequency and the phase-shift of the waveform. Moreover, the correlation between two distinct waveforms, $s_{m, i}(t)$ and $s_{m^{\prime}, j}(t)$ is given as:

$$
\left\langle s_{m, i}, s_{m^{\prime}, j}\right\rangle=\left\{\begin{array}{ll}
\frac{2}{3} E_{\mathrm{s}_{(\mathrm{elec})}}\left[1+\frac{1}{2} \cos \left(\frac{2 \pi}{M_{\varphi}}(i-j)\right)\right] & m=m^{\prime} \\
\frac{2}{3} E_{\mathrm{s}_{(\mathrm{elec})}} & m \neq m^{\prime}
\end{array},\right.
$$

where $\langle\cdot, \cdot\rangle$ indicates the dot product. (3) implies that inclusion of $\beta$ contributes to a loss of orthogonality which prevailed for the AC component of the waveforms (as in $M$-ary FSK [2]).

Fig. 1 depicts a generalized transmitter architecture for $\left(M_{\perp}, M_{\varphi}\right)$-DC-FPSK. A pragmatic implementation approach is to generate a dictionary, $\mathcal{D}$ of all possible waveforms, which takes into consideration $m, \varphi_{i}$ and $\beta$, and interpolate it in a look-up-table (LUT). For a given $M_{\perp}$ and $M_{\varphi}$, the cardinality of $\mathcal{D}$, i.e., $|\mathcal{D}|=M_{\perp} M_{\varphi}$. For disambiguation, we shall call the discrete-time representation of the waveform a symbol, and express it with boldface lowercase letters. For discrete-time symbol generation, $M_{\perp}$ positive discrete frequencies lead to $2 M_{\perp}+1$ points in the (negative, null and positive) frequency grid, which requires a minimum of $M_{\mathrm{c}}=2 M_{\perp}+1$ number of chips per symbol, i.e., $T_{\mathrm{S}} / T_{\mathrm{c}}=2 M_{\perp}+1$. In practice, the use of Fast Fourier Transform (FFT) with a higher number of chips (e.g., $4 M_{\perp}$ ) can be used for efficient oversampling implementation.

\section{B. Maximum Likelihood Recovery}

Considering a priori equiprobability of transmit symbols, i.e., $p\left(s_{m, i}\right)=1 / M_{\perp} M_{\varphi}$, the $\mathrm{ML}$ criterion dictates to identify $\boldsymbol{s}_{m, i}$ in $\mathcal{D}$, which maximizes the likelihood function, $p\left(\boldsymbol{r} \mid \boldsymbol{s}_{m, i}\right) . p\left(\boldsymbol{r} \mid \boldsymbol{s}_{m, i}\right)$ is the conditional probability density function of receiving $\boldsymbol{r}$ when $\boldsymbol{s}_{m, i}$ is sent. Since $\beta$ does not influence the likelihood function, hence, $p\left(\boldsymbol{r} \mid \boldsymbol{s}_{m, i}\right)=$ $p\left(\tilde{\boldsymbol{r}} \mid \tilde{\boldsymbol{s}}_{m, i}\right)$, where $\tilde{\boldsymbol{r}}=\boldsymbol{r}-\beta$, and $\tilde{\boldsymbol{s}}_{m, i}=\boldsymbol{s}_{m, i}-\beta$. Considering an AWGN channel, with variance $\sigma_{\mathrm{ch}}^{2}=N_{0} B$, the likelihood function is given as [7]:

$$
\begin{aligned}
p\left(\tilde{\boldsymbol{r}} \mid \tilde{\boldsymbol{s}}_{m, i}\right) & =\left(1 / 2 \pi \sigma_{\mathrm{ch}}^{2}\right)^{M_{\mathrm{c}}} \exp \left(-\left\|\tilde{\boldsymbol{r}}-\tilde{\boldsymbol{s}}_{m, i}\right\|^{2} / 2 \sigma_{\mathrm{ch}}^{2}\right) \\
& =\mathrm{C}_{\mathrm{st}} \exp \left(\left\langle\tilde{\boldsymbol{r}}, \tilde{\boldsymbol{s}}_{m, i}\right\rangle / \sigma_{\mathrm{ch}}^{2}\right) .
\end{aligned}
$$

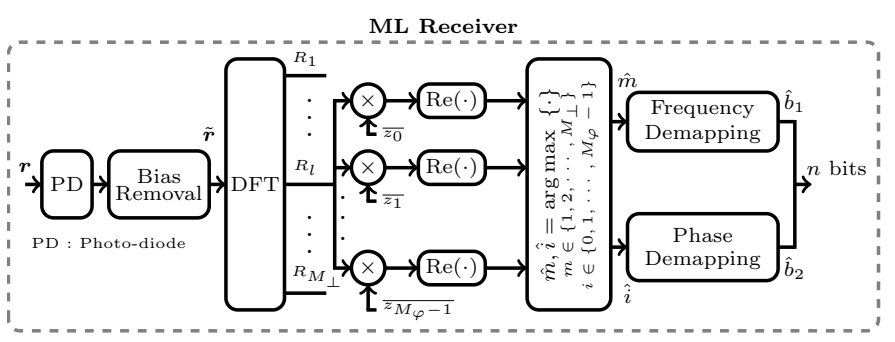

Fig. 2: Receiver structure of $\left(M_{\perp}, M_{\varphi}\right)$-DC-FPSK corresponding to (8).

\|. $\|$ evaluates the Euclidean norm and $\mathrm{C}_{\mathrm{st}}=$ $\left(1 / 2 \pi \sigma_{\mathrm{ch}}^{2}\right)^{M_{\mathrm{c}}} \exp \left(-\left(\|\tilde{\boldsymbol{r}}\|^{2}+\left\|\tilde{\boldsymbol{s}}_{m, i}\right\|^{2}\right) / 2 \sigma_{\mathrm{ch}}^{2}\right) . \quad\|\tilde{\boldsymbol{r}}\|^{2} \quad$ is constant, and for any $m$ and $i,\left\|\tilde{\boldsymbol{s}}_{m, i}\right\|^{2}=E_{\mathrm{s}_{(\mathrm{elec})}} / 3$. The estimated symbols under ML criterion are:

$$
\hat{\boldsymbol{s}}_{\hat{m}, \hat{i}}=\underset{\tilde{\boldsymbol{s}}_{m, i} \in \tilde{\mathcal{D}}}{\arg \max } p\left(\tilde{\boldsymbol{r}} \mid \tilde{\boldsymbol{s}}_{m, i}\right)=\underset{\tilde{\boldsymbol{s}}_{m, i} \in \tilde{\mathcal{D}}}{\arg } \max \left\langle\tilde{\boldsymbol{r}}, \tilde{\boldsymbol{s}}_{m, i}\right\rangle,
$$

where $\tilde{\mathcal{D}}$ is the dictionary of unbiased waveforms, $\tilde{\boldsymbol{s}}_{m, i}$. This naive ML correlation receiver requires $M_{\mathrm{C}} M_{\perp} M_{\varphi}$ (real) multiplications.

Proposition 1: $M_{\perp} \times M_{\varphi}$ segments of $\left\langle\tilde{\boldsymbol{r}}, \tilde{\boldsymbol{s}}_{m, i}\right\rangle$ can be appraised by evaluating the real components of $M_{\perp}$ segments of $M_{\mathrm{c}}$ order DFT of $\tilde{\boldsymbol{r}}$ followed by $M_{\varphi}$ phase-shifts.

Proof: The real-valued discrete-time symbol, $\tilde{s}_{m, i}(k)$ can be rewritten using complex notation as:

$$
\tilde{s}_{m, i}(k)=A \cos \left(2 \pi m k \Delta f T_{\mathrm{c}}+\varphi_{i}\right)=\operatorname{Re}\left(z_{i} f_{m}(k)\right),
$$

for $k=\left\{0,1, \cdots, M_{\mathrm{c}}-1\right\}$, where $z_{i}=\exp \left(j \varphi_{i}\right)$ are the phase-shifts in complex plane and $j=\sqrt{-1} . f_{m}(k)=$ $A \exp \left(j 2 \pi m \Delta f k T_{\mathrm{c}}\right)$ defines the pure tone at the selected frequency. Accordingly, $\left\langle\tilde{\boldsymbol{r}}, \tilde{\boldsymbol{s}}_{m, i}\right\rangle$ is given by

$$
\begin{aligned}
\left\langle\tilde{\boldsymbol{r}}, \tilde{\boldsymbol{s}}_{m, i}\right\rangle & =\sum_{k=0}^{M_{\mathrm{c}}-1} \tilde{r}(k) \overline{\tilde{s}_{m, i}(k)}=\sum_{k=0}^{M_{\mathrm{c}}-1} \tilde{r}(k) \operatorname{Re}\left(\overline{z_{i} f_{m}(k)}\right) \\
& =\operatorname{Re}\left(\overline{z_{i}} \sum_{k=0}^{M_{\mathrm{c}}-1} \tilde{r}(k) \overline{f_{m}(k)}\right)=\operatorname{Re}\left(\overline{z_{i}}\left\langle\tilde{\boldsymbol{r}}, \boldsymbol{f}_{m}\right\rangle\right),
\end{aligned}
$$

where overline denotes the conjugate. $R_{m}=\left\langle\tilde{\boldsymbol{r}}, \boldsymbol{f}_{m}\right\rangle$ represents the $m$ th segment of the DFT of $\tilde{\boldsymbol{r}}$. In the sequel, we shall only appraise $M_{\perp}$ segments among $M_{\mathrm{c}}$, which are grouped in $\boldsymbol{R}=\left[R_{1}, R_{2}, \cdots, R_{M_{\perp}}\right]^{\mathrm{T}}$.

According to (7), the decision on the transmitted frequency is made using $\boldsymbol{R}$ and phase-shifts, $z_{i}$ as:

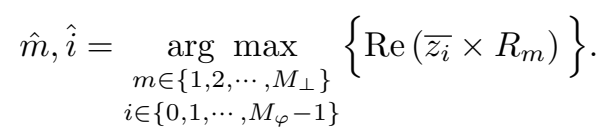

The receiver structure conforming to (8) is presented in Fig. 2. With this implementation, the (real) multiplications are reduced to $M_{\perp}\left(2 M_{\mathrm{c}}+4 M_{\varphi}\right)$.

\section{Reduced Complexity Sub-optimal Receiver}

A reduced complexity sub-optimal receiver for $\left(M_{\perp}, M_{\varphi}\right)$ DC-FPSK is illustrated in Fig. 3. $\boldsymbol{R}$ obtained via DFT of $\tilde{\boldsymbol{r}}$, encodes both the frequency and the phase-shift of transmitted $\left(M_{\perp}, M_{\varphi}\right)$-DC-FPSK waveform. Thus, using $\boldsymbol{R}$, the transmit 
Sub-optimal Receiver

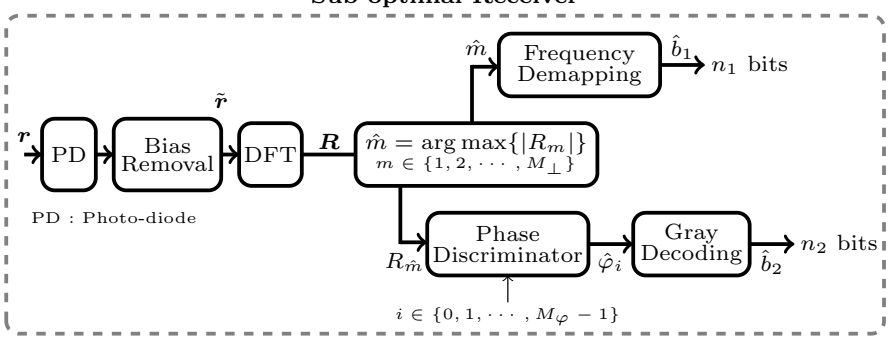

Fig. 3: Sub-optimal receiver structure of $\left(M_{\perp}, M_{\varphi}\right)$-DC-FPSK.

frequency is identified as:

$$
\hat{m}=\underset{m \in\left\{1,2, \cdots, M_{\perp}\right\}}{\arg \max }\left\{\left|R_{m}\right|\right\},
$$

Moreover, the estimated phase of the received waveform, $\hat{\varphi}_{i}$ is determined by decoding $R_{\hat{m}}$ into the phase discriminator. The bits encoded into $\hat{\varphi}_{i}$ are retreived via Gray decoding.

\section{Analytical and Simulation Results}

In this section, we provide the analytical analysis of spectral efficiency and minimum squared Euclidean distance (MSED) for the proposed approach. Moreover, Monte Carlo simulation results are provided for BER evaluation and to discern the evolution of BER with respect to the spectral efficiency.

\section{A. Spectral Efficiency Analysis}

$\left(M_{\perp}, M_{\varphi}\right)$-DC-FPSK transmits $n_{1}+n_{2}=\log _{2}\left(M_{\perp} M_{\varphi}\right)$ bits per symbols, proceeding in a data-rate of $R=$ $\log _{2}\left(M_{\perp} M_{\varphi}\right) / T_{\mathrm{s}}$ and the SE, $\eta$ of

$$
\eta=\frac{R}{B}=\frac{\log _{2}\left(M_{\perp} M_{\varphi}\right)}{M_{\perp}} \quad(\text { bits } / \mathrm{s} / \mathrm{Hz}) .
$$

(10) signifies that $\eta$ augments by increasing $M_{\varphi}$ and keeping $M_{\perp}$ constant. Conversely, if $M_{\varphi}$ is kept constant and $M_{\perp}$ is increased, $\eta$ tends to diminish.

\section{B. Euclidean Distance Analysis}

The squared Euclidean distance, $d_{m, m^{\prime}}^{2}=\| s_{m, i}(t)-$ $s_{m^{\prime}, j}(t) \|^{2}$ is given as:

$$
d_{m, m^{\prime}}^{2}=2 E_{\mathrm{s}_{(\mathrm{elec})}}-2\left\langle s_{m, i}(t), s_{m^{\prime}, j}(t)\right\rangle .
$$

By incorporting (3) in (11), $d_{m, m^{\prime}}^{2}$ becomes

$$
d_{m, m^{\prime}}^{2}= \begin{cases}\frac{2}{3} E_{\mathrm{s}_{(\mathrm{elec})}}\left[1-\cos \left(\frac{2 \pi}{M_{\varphi}}(i-j)\right)\right] & m=m^{\prime} \\ \frac{2}{3} E_{\mathrm{s}_{(\mathrm{elec})}} & m \neq m^{\prime}\end{cases}
$$

resulting in MSED, $d_{\min }^{2}$ of

$$
d_{\min }^{2}=\left\{\begin{array}{ll}
\frac{2}{3} E_{\mathrm{s}_{(\mathrm{elec})}} & M_{\varphi}=2,4 \\
\frac{2}{3} E_{\mathrm{s}_{(\mathrm{elec})}}\left[1-\cos \left(\frac{2 \pi}{M_{\varphi}}\right)\right] & M_{\varphi}>4
\end{array} .\right.
$$

(13) delineates that for a given $E_{\mathrm{b}_{\text {(elec) }}}$, increasing $M_{\perp}$ increases $d_{\text {min }}^{2}$ as $E_{\mathrm{s}_{(\text {elec })}}=E_{\mathrm{b}_{(\text {elec })}} \log _{2}\left(M_{\perp} M_{\varphi}\right)$. Conversely, even though $E_{\mathrm{s}_{(\mathrm{elec})}}$ increases with $M_{\varphi}$ but overall $d_{\text {min }}^{2}$

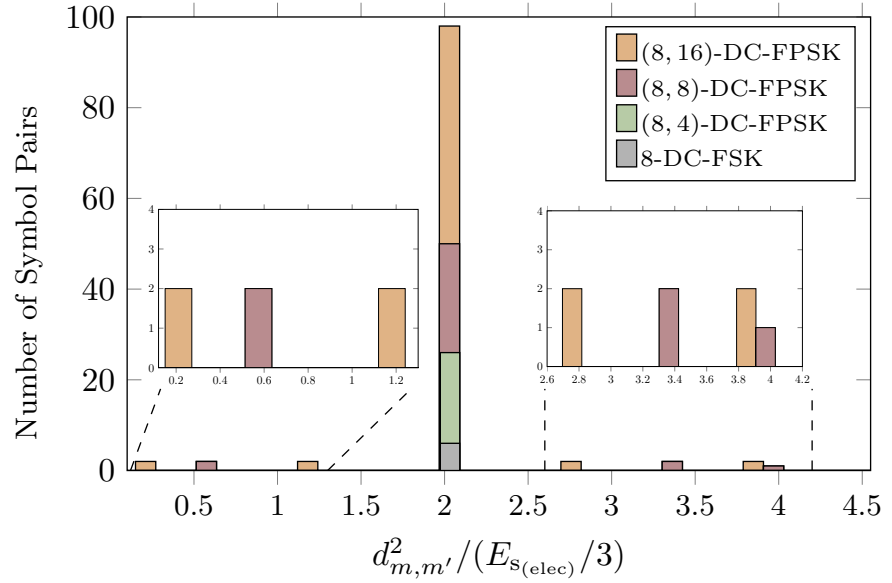

Fig. 4: Histogram of the squared normalized Euclidean distances, $d_{m, m^{\prime}}^{2}$, obtained using analytical result in (12).

decreases. Fig. 4 illustrates simulated $d_{m, m^{\prime}}^{2}$ obtained using $M_{\perp}=8$ and varying $M_{\varphi}$. It may be noticed that changing $M_{\varphi}$ from 4 to 8 reduces $d_{\text {min }}^{2} / E_{\mathrm{s}_{(\mathrm{elec})}}$ from 0.667 to approximately 0.197 . Moreover, we can also observe that $d_{\min }^{2} / E_{\mathrm{s}_{(\mathrm{elec})}}$ of $\left(M_{\perp}, M_{\varphi}\right)$-DC-FPSK is same as that of $M$-ary DC-FSK (with $\left.M=M_{\perp}\right)$ when $M_{\varphi} \leq 4$.

\section{Bit Error Rate Performance}

In this section, we discuss the simulated BER performance of the $\left(M_{\perp}, M_{\varphi}\right)$-DC-FPSK. We use $M$-ary DC-FSK with $M=M_{\perp}$, OOK and $M$-ary PAM as references. For a first proof-of-concept, we consider an AWGN channel for comparison as conventionally done in the literature [9]. The results are averaged over $3 \times 10^{4}$ runs.

The BER of $\left(M_{\perp}, M_{\varphi}\right)$-DC-FPSK not only depends on $d_{\text {min }}^{2}$, but also on the number of symbol pairs separated by $d_{\text {min }}$. Fig. 4, identifies that $M_{\varphi} \leq 4$ should be a priori prefered because of highest MSED. Though, for $M_{\varphi}>4$, $d_{\text {min }}$ decreases, but, the number of symbol pairs exhibiting $d_{\text {min }}$ remains limited. Thus, it is not trivial to predict the impact of change in $M_{\varphi}$ on BER. We determine (Fig. 5) that $M_{\varphi}=4$ persistently achieves the optimal performance in terms of required $E_{\mathrm{b}_{(\text {elec })}} / N_{0}$ to attain a given BER. Fig. 6 depicts the BER performance of $\left(M_{\perp}, M_{\varphi}\right)$-DC-FPSK using $M_{\perp}=\{8,16\}$ and $M_{\varphi}=4$. It may be observed that $\left(M_{\perp}, M_{\varphi}\right)$-DC-FPSK not only provides high $\mathrm{SE}$ but is also energy efficient compared to $M$-ary DC-FSK. Both the ML and the sub-optimal receivers manifest better performance than $M$-ary DC-FSK. On the other hand, the BER trend of $\left(M_{\perp}, M_{\varphi}\right)$-DC-FPSK is in complete contradiction with OOK and $M$-ary PAM, as it becomes energy efficient for higher alphabet cardinalities.

Fig. 7 depicts EE versus SE performance of $\left(M_{\perp}, 4\right)$-DCFPSK for both ML and the sub-optimal receiver for a target BER of $10^{-3} . M_{\perp}=\{4,8,16,32,64,128,256,512,1024\}$ is used to vary $\eta$. We observe that, the sub-optimal receiver requires marginally increased $E_{\mathrm{b}_{\text {(elec) }}} / N_{0}$ compared to the ML receiver. We also observe that for smaller $M_{\perp}$ (and $M_{\varphi}=4$ ), the improvement in the EE for DC-FPSK is substantial compared to $M$-ary DC-FSK. However, for higher 

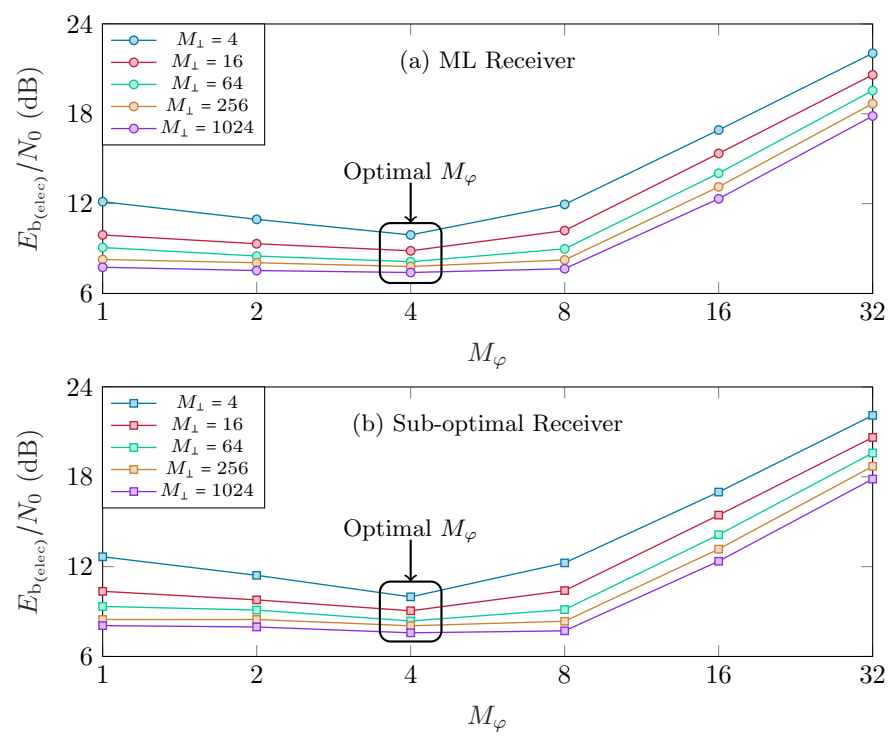

Fig. 5: Evaluation of optimum $M_{\varphi}$ for different $M_{\perp}$ to achieve a BER of $10^{-3}$ : (a) ML Receiver; (b) Sub-optimal Receiver.

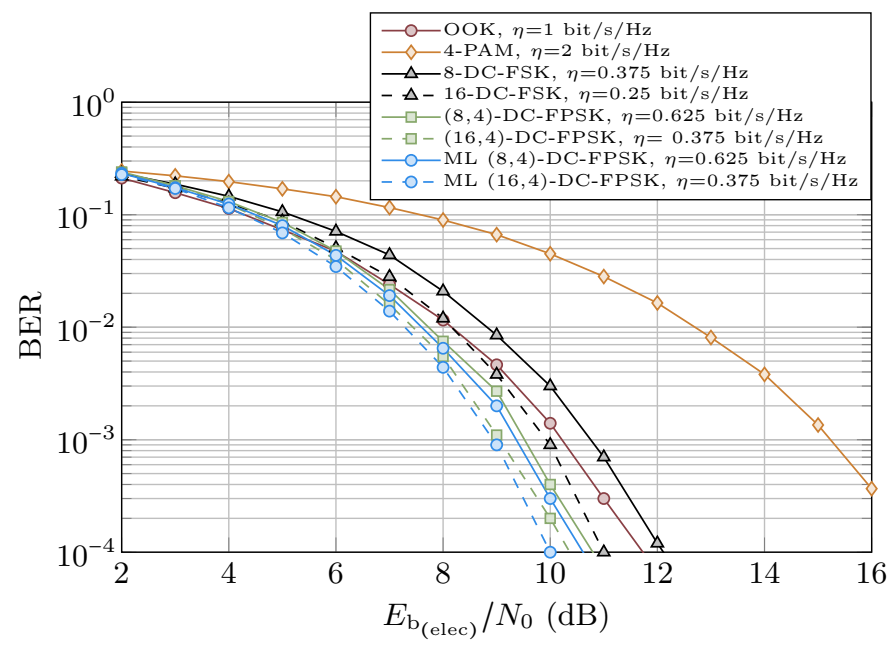

Fig. 6: Simulation results for BER performance of $\left(M_{\perp}, M_{\varphi}\right)$-DC-FPSK. The abbreviation 'ML' refers to ML receiver, whereas, for sub-optimal receiver, no abbreviation has been used. $\eta$ for $M$-ary PAM is evaluated as $\log _{2}(M)$.

$M_{\perp}$, the gain becomes less noticable due to marginal increase in SE. Thence, we distinguish a region of interest (ROI) (illustrated in Fig. 7) which determines the range of spectral efficiencies that may be targeted for DC-FPSK. Apart from that, unlike $M$-ary PPM, $\left(M_{\perp}, M_{\varphi}\right)$-DC-FPSK has a constant envelop and manifests a limited PAPR of approximately $3 \mathrm{~dB}$.

\section{Example of Practical Parameters}

To highlight the practicality of $\left(M_{\perp}, M_{\varphi}\right)$-DC-FPSK, we evaluate $T_{\mathrm{s}}, T_{\mathrm{c}}, \Delta f$ and $B$ by fixing $R=1$ Mbits/s [5] and $M_{\varphi}=4$. We choose $M_{\perp}=128$ which corresponds to the worst case scenario in the ROI. For implementation, the following values of the parameters are needed: $T_{\mathrm{s}}=9 \mu \mathrm{s}$, $1 / T_{\mathrm{c}} \approx 28.55 \mathrm{Mchips} / \mathrm{s}, \Delta f \approx 11.11 \mathrm{kHz}$ and $B \approx 14.22$ $\mathrm{MHz}$. For the corresponding spectral efficiency, i.e., $\eta \approx 0.07$ $\mathrm{bit} / \mathrm{s} / \mathrm{Hz}$, the chip time needed is higher compared to data rate $R$, but still remains a relaxed requirement for modern

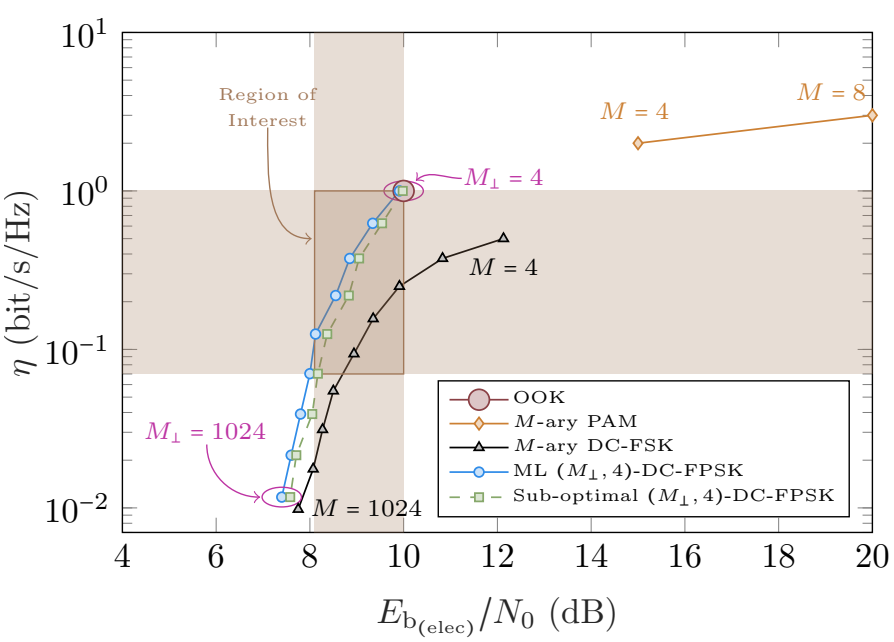

Fig. 7: Evolution of required $E_{\mathrm{b}}$ (elec) $/ N_{0}$ with respect to $\eta$ to achieve a BER of $10^{-3}$ obtained via Monte Carlo simulations.

converters. Furthermore, $B$ lies well within the range of LED bandwith, which is a few tens of $\mathrm{MHz}$.

\section{CONCLUSIONS}

$\left(M_{\perp}, M_{\varphi}\right)$-DC-FPSK capitalizes on the benefits of $M$-ary DC-FSK and adds flexibility in terms of SE by introducing phase-shifts in the transmit waveforms. We comprehensively elucidate $\left(M_{\perp}, M_{\varphi}\right)$-DC-FPSK waveform generation and recovery using ML and sub-optimal receivers. MSED analysis identifies that $M_{\varphi}=4$ may provide the optimal performance as it increases the SE without reducing the MSED. Moreover, $\left(M_{\perp}, M_{\varphi}\right)$-DC-FPSK enhances the $\mathrm{SE}$ and EE compared to $M$-ary DC-FSK. Contrary to $M$-ary PAM, the EE of $\left(M_{\perp}, M_{\varphi}\right)$-DC-FPSK is closer to the Shannon's EE limit for high $M_{\perp}$. The advantages over classical approaches identified hereby makes $\left(M_{\perp}, M_{\varphi}\right)$-DC-FPSK a viable candidate for IoT based on OWS.

\section{REFERENCES}

[1] H Park and J. R. Barry. Trellis-coded multiple-pulse-position modulation for wireless infrared communications. IEEE Trans. Commun., 52(4):643651, 2004.

[2] K. Kikuchi and M. Osaki. Highly-sensitive coherent optical detection of M-ary frequency-shift keying signal. Opt. Express, 19(26):B32-B39, 2011.

[3] S. Arnon. The effect of clock jitter in visible light communication applications. IEEE/OSA J. Light. Technol., 30(21):3434-3439, 2012.

[4] S. He, G. Ren, Z. Zhong, and Y. Zhao. M-ary variable period modulation for indoor visible light communication system. IEEE Commun. Lett., 17(7):1325-1328, 2013.

[5] A. W. Azim, A. Rullier, Y. Le Guennec, L. Ros, and G. Maury. Energy efficient $M$-ary frequency-shift keying based modulation techniques for visible light communication. IEEE Trans. Cogn. Commun. Netw., (Early Access), 2019

[6] G.M.M. Yamga, A.R. Ndjiongue, and K. Ouahada. Low complexity clipped frequency shift keying (FSK) for visible light communications. In IEEE Intl. Conf. Adapt. Sci. \& Techn., pages 1-6. IEEE, 2018.

[7] Y. Roth, J.-B. Doré, L. Ros, and V. Berg. Coplanar Turbo-FSK: A flexible and power efficient modulation for the Internet-of-Things. Wirel. Commun. Mob. Com., 2018.

[8] R. A. Khalona, G. E. Atkin, and J. L LoCicero. On the performance of a hybrid frequency and phase shift keying modulation technique. IEEE Trans. Commun., 41(5):655-659, 1993.

[9] J. Armstrong and B. J. C. Schmidt. Comparison of asymmetrically clipped optical OFDM and DC-biased optical OFDM in AWGN. IEEE Commun Letters, 12(5):343-345, 2008. 